\title{
ALBERT LAUTMAN ET LE SOUCI BRISÉ DU MOUVEMENT
}

\author{
Charles AlunNi
}

RÉSUMÉ : Nous posons l'œuvre d'Albert Lautman comme une sorte d'opérateur de brisure de symétrie dans le cadre de l'opposition traditionnelle de la philosophie spéculative et des sciences physico-mathématiques. L'enjeu pour la philosophie en est, à de très rares exceptions près, encore très mal perçu. Sur ce plan, nous reprenons la question du «platonisme » supposé de Lautman, et nous le confrontons à sa lecture fondamentale de Martin Heidegger. Les conséquences de cette inscription dans le sillon heideggérien sont fondamentales pour une juste restitution de cette pensée et de cette philosophie dans ce qu'elles conservent de plus actuel. Sur l'autre versant, celui des percées lautmaniennes dans le champ de la physique mathématique, nous insistons sur l'extrême alacrité prospectiviste de son regard, sa puissance philosophique venant renforcer cette singulière sensibilité à ce qui fit l'essentiel des mathématiques et de la physique théorique de son temps. En ce sens, sa position occupe bel et bien l'un des points nodaux du site « surrationaliste ».

MoTS-CLÉS : épistémologie, métaphysique, schéma, structure, dualité, involution, problème, différence ontologique, symétrie, Heidegger.

ABSTRACT : We locate Albert Lautman's work as a sort of agent of the break of symmetry in the framework of the traditional opposition of speculative philosophy and the physico-mathematical sciences. The stakes for philosophy are, with very rare exceptions, very poorly perceived. On this level, we return to the question of Lautman's supposed «Platonism » and we confront it with its fundamental reading of Martin Heidegger. The consequences of this insertion in the heideggerian furrow are fundamental for a just restitution of this thought and this philosophy into what they retain in current thought. On the other side, that of Lautmanian entries in the field of mathematical physics, we insist on the extreme prospective alacrity of his gaze, his philosophic power which comes to reinforce this strange sensibility toward what constituted the essential part of theoretical mathematics and physics in his time. In this sense, his position occupies actually one of the nodal points of the «surrationalistic » landscape.

KEYWORDS : epistemology, metaphysics, scheme, structure, duality, involution, problem, ontological difference, symmetry, Heidegger. 
ZuSAMmENFASSUNG : Wir positionieren das Werk von Albert Lautman wie einen Symmetriebrechungsoperator in den Rahmen des traditionellen Gegensatzes zwischen spekulativer Philosophie und physikalisch-mathematischer Wissenschaft. Der mögliche Gewinn für die Philosophie ist, von sehr wenigen Ausnahmen abgesehen, noch kaum erkannt worden. Wir nehmen Lautmans Ausgangsfrage des «Platonismus » auf und stellen sie seiner gründlichen Lektüre von Martin Heidegger gegenüber. Eine solche Inskription in die Heideggersche Spur hat fundamentale Folgen für eine angemessene Rekonstruktion dessen, was in diesem Denken und dieser Philosophie an Aktuellem bewahrt ist. Auf der anderen Seite - der der Lautmanschen Durchbrüche auf dem Feld der mathematischen Physik - betonen wir die extreme vorausschauende Bereitwilligkeit seines Blickes : sein philosophisches Vermögen hat diese einzigartige Sensibilität für das Essentielle in der Mathematik und theoretischen Physik seiner Zeit verstärkt. In diesem Sinn nimmt seine Position sehr wohl einen der Knotenpunkte der « surrationalistischen » Szenerie ein.

STICHWÖRTER : Epistemologie, Metaphysik, Schema, Struktur, Dualität, Involution, Problem, ontologische Differenz, Symmetrie, Heidegger.

Charles AlunNI, né en 1951, fut cofondateur et secrétaire technique du Groupe de recherches sur l'enseignement de la philosophie, fondé à l'École normale supérieure de Paris (rue d'Ulm) en 1975 à l'initiative de Jacques Derrida. Directeur de programme au Collège international de philosophie de 1983 à 1989 et Gastdozent au département de philosophie de l'université de Bochum (Allemagne) de 1983 à 1987, il est actuellement chargé de recherche en histoire de la philosophie à l'École normale supérieure de Pise (Italie), et professeur détaché à l'École normale supérieure de Paris, où il dirige depuis 1994 le Laboratoire disciplinaire « Pensée des sciences ».

Adresse : École normale supérieure, Laboratoire disciplinaire «Pensée des sciences », 45 rue d'Ulm, 75230 Paris cedex 05.

Courrier électronique : Charles.Alunni@ens.fr 
À Jacques Derrida, l'ami, À celui dont l'influence spectrale ne se laissera jamais mesurer ni la perte réparer.

PRÉAMBULE

Dijon, 11 Janvier 1938

\section{Cher Monsieur,}

Je vous remercie bien vivement pour l'envoi de vos thèses et pour les dédicaces qui me touchent vivement. Je viens seulement d'en achever la lecture et je suis bien frappé par la richesse des aperçus. À toutes les pages on trouve matière à réflexion. En évinçant les calculs, vous avez su garder la pensée. Et cela n'est pas un mince mérite. Tout philosophe scientifique sait que c'est là une difficulté presque insurmontable. Malheureusement, le lecteur philosophe ne s'en doute pas, et il accuse l'auteur d'obscurité plutôt que de s'accuser d'ignorance. Mais il faut que nous changions tout cela. Avec quelle sympathie je salue en vous un représentant de la jeune équipe qui va ramener la philosophie aux tâches héroïques de la pensée difficile. Si nous pouvions être une dizaine ! Ne manquez pas de me signaler vos élèves, vos disciples, car vous allez bientôt avoir des disciples. Dans la même estime, je tiens Cavaillès. Je n'ai pas encore de nouvelle de sa thèse. Il ne va pas tarder je pense à la soutenir. Si vous le voyez, faites-lui mes amitiés.

Si vous n'avez personne aux Recherches philosophiques de l'an prochain pour faire un compte rendu, donnez mon nom à Koyré ou à Wahl, et je me chargerai très volontiers de ce compte rendu qui me permettra de dire publiquement tout le bien que je pense de votre travail.

Vers la fin du mois, je compte vous envoyer mon nouveau livre. C'est un livre de détente, écrit en marge d'interminables lectures $[\ldots]^{1}$.

Le destinataire de cette lettre est Albert Lautman ; quant à son auteur, ce n'est autre que Gaston Bachelard, et l'ouvrage en question (« livre de détente »), La Formation de l'esprit scientifique, publié en $1938^{2}$ ! On pourrait dire que tout est dit - ou presque -, et que ce dire en dit aussi beaucoup sur son auteur, sur son éthique et sur sa philosophie (celle des « philosophes scientifiques »), sur son appel impératif à l'action - « [...] il faut que nous changions tout cela »-, sur son sentiment de « solitude » dans la nécessaire endurance de la pensée (qualifiée de « difficile ») : « Si nous pouvions être une dizaine ! »

Plus que tout, c'est ici un «envoi », une « injonction », lancés vers l'avenir qui ne cesse encore d'inquiéter notre présent, ici et maintenant, en ces murs, dans ce colloque

1. BACHELARD, in BENIS-SINACEUR, $1987 \mathrm{~b}$.

2. Voir BACHELARD, 1938. 
suisse, toujours habité par quantité de « spectres » revenant inlassablement. Cette revenance sonne comme en un étrange écho, par la bouche même de Bachelard qui ne pouvait savoir, «raisonnablement », ce que le 17 mai précédent Jean Cavaillès avait écrit d'Amiens à son camarade Albert Lautman : « Il est heureux que nous soyons deux dans l'ingrat pays de la philosophie des sciences ${ }^{3}$. » Ajoutons également l'ombre anticipée d'une autre spectralité, sinistre celle-là, dont Cavaillès fera part à Lautman en septembre 1938 : «Cher ami, [j]e suis à Paris depuis hier matin. Le congrès [d'Amersfoort en Hollande] s'est effondré samedi : dans le brouillard hollandais, toutes les ombres devenaient énormes ${ }^{4}$. » « Nuit et brouillard »-déjà consommés. C'est là que Cavaillès y rencontra Alfred Tarski et Ferdinand Gonseth, au cours d'entretiens habités par la chape d'angoisses des prémices de guerre, comme nous l'a rapporté notre amie, présente à Amersfoort, Paulette Destouches-Février.

Le décor est planté, et déjà notre discours paraît tenir de la gageure en prétendant traiter le contenu de son titre. D'où la nécessité de présenter l'axiomatique minimale de deux œuvres indéfectiblement tressées par les nœuds de leur brin. Nous polariserons donc l'analyse sur quatre focales explicites, au risque de l'objection de trop grande densité et de trouble opacité. Nous nous voyons donc contraint de répondre à l'objection, surtout en cette époque où le temps n'a plus le temps que de compter sur l'immédiateté des compréhensions mystificatrices par «survols », par ce que Georges Canguilhem et Charles Ehresman notaient à propos des écrits de Cavaillès, avec la violence toujours nécessaire de toute revendication philosophique et scientifique : «Ceux qui ne feront pas l'effort nécessaire pour comprendre ne méritent pas d'être éclairés ${ }^{5}$. »

En octobre 1984, Bruno Huisman déclarait à propos de Cavaillès :

«Soyons honnêtes, ou du moins réaliste: on peut être, aujourd'hui, professeur de philosophie sans avoir jamais lu une seule ligne de Cavaillès. Invoquée souvent, citée parfois, l'œuvre de Cavaillès est peu fréquentée pour elle-même ${ }^{6}$. »

En ce qui concerne la figure d'Albert Lautman, force est de constater que la situation est encore pire. Car si en 1994 l'éditeur Hermann, sous l'impulsion de Huisman et de Canguilhem, recueillait la quasi-totalité des textes de Cavaillès en un épais volume des Cuvres complètes de philosophie des sciences, l'Essai sur l'unité des mathématiques et divers écrits de Lautman, paru à l'Union générale d'éditions en 1977, épuisé avant les années 1980, n'a quant à lui jamais été réédité ! Cela restera pour nous comme un des plus gros scandales de l'édition française. Ainsi, Jean Petitot peut à juste titre affirmer :

«Considérée comme trop spéculative, malgré son exceptionnelle érudition mathématique et son rapport étroit au structuralisme axiomatique hilbertien, sa philosophie mathématique n'a, jusqu'ici, fait l'objet d'aucune attention particulière [...]. Pour le dire d'emblée, Albert Lautman représente selon nous, sans emphase, un des philosophes les plus inspirés de ce siècle ${ }^{7}$. »

3. Cavaillès, in Benis-Sinaceur, 1987a, p. 122.

4. CAVAillès, in Benis-Sinaceur, 1987a, p. 122. Dans les citations, sauf mention contraire, c'est nous qui soulignons.

5. Canguilhem et Ehresman, 1947, in Cavaillès, 1994, p. 482.

6. Huisman, 1985, p. 25.

7. Ретітот, 1987, p. 79-80. 
On pourrait rendre raison, et même et surtout « raison philosophique » et « épochale » de cette sinistre situation, mais nous ne le ferons pas ici. Reconnaissons simplement qu'il y a une réputation légitime de difficulté, voire même de grande austérité, attachées au binôme Cavaillès-Lautman - et c'est précisément tout ce qui en fait le prix, gage de leur importance, tant pour leur présent que pour notre avenir. La constitution d'une « nouvelle philosophie mathématique » présuppose une ascèse laborieuse. Le jugement bachelardien porté sur Cavaillès, s'étend (et s'entend) également à (et de) Lautman : « Ainsi, on ne peut trouver, dans les œuvres de notre ami, aucun préambule de lente initiation, aucun aperçu de facile généralité, aucune préparation psychologique élémentaire. Pour lire Cavaillès, il faut travailler $^{8}$. » Leur souci logique (et commun) aura toujours été de saisir, dans leur autonomie, les gestes constructeurs de la mathématique effective. Or, ce n'est qu'une fois « installés dans le champ » que l'on peut être en mesure d'élaborer, à nouveaux frais, une doctrine de la pensée elle-même : « La connaissance mathématique est centrale pour savoir ce qu'est la connaissance ${ }^{9}$. » L'installation dans le champ correspond pour Cavaillès au défrichage de la théorie abstraite des ensembles et à la découverte d'un « nouveau monde » (suivant la formule de Bartel Leendert Van der Waerden) : le monde de la «mathématique des algébristes » dont le centre mondial se situe alors à Göttingen ; quant à Lautman, l'humus de ses recherches épistémologiques comprendra non seulement l'œuvre de son ami Jacques Herbrand, mais également celle de David Hilbert (avec sa théorie du corps de classes et les espaces du même nom), la Moderne algebra de Van der Waerden ${ }^{10}$, la topologie algébrique développée par Paul Alexandroff, Heinz Hopf et Lev Seminovich Pontrjagin, la théorie des groupes quantiques d'Hermann Weyl, les travaux d'Élie Cartan (sur les espaces symétriques et l'algèbre extérieure), enfin l'opération de re-fondation de Nicolas Bourbaki (à travers Claude Chevalley et Henri Cartan).

Du silence pesant concernant le travail philosophique de Lautman auprès des philosophes contemporains, une exception cependant (en dehors, évidemment, de philosophes des sciences ou de scientifiques philosophes comme Jean-Toussaint Desanti, Dominique Lambert, Jean Petitot ou Gilles Châtelet ${ }^{11}$ ) : celle de Gilles Deleuze dès 1968, dans Différence et répétition ${ }^{12}$. Ses citations incitatives à une lecture attentive portent sur les

8. BACHELARD, 1950, p. 223.

9. CAVAILlÈs, « La pensée mathématique », séance du 4 février 1939 à la Société française de philosophie, Bulletin de la Société française de philosophie, in CAVAILLÈs, 1994, p. 583-630.

10. VAN DER WAERDEN, 1930.

11. Et bien évidemment, d'Alain Badiou : «Je dois ici déclarer que les écrits de Lautman sont proprement admirables, et que ce que je leur dois, jusque dans les intuitions fondatrices de ce livre, ne se laisse pas mesurer (BADIOU, 1988, p. 522, notes des pages 18 et 19). Il convient d'ajouter les occurrences suivantes: BADIOU, 1992, p. 158 (note 26) ; BADIOU, 1989, p. 83-84 ; BADIOU, 1997, p. 144 ; BADIOU, 1998a, p. 13, 14 et 17; BADIOU, 1998b, p. 56.

12. DeLEuZE, 1968, p. 212-213 et 230-238. En réalité, la référence lautmanienne parcourt l'ensemble de l'œuvre deleuzienne. Par exemple : Deleuze, 1969, p. 32, 69 et 127 ; Deleuze, 1980, p. 462 et 606-607 ; DeleuZe, 1986, p. 85 ; DeleuZe, 1988, p. 136. Sur l'influence de cette référence deleuzienne, nous renvoyons ici au témoignage du philosophe des mathématiques Jean-Michel Salanskis : «Cette convocation des mathématiques par Deleuze m'a toujours semblé, dans son principe, légitime et correcte. J'ajouterai même que la lecture de Deleuze m'a incité à lire Lautman, ce dont je ne lui serai jamais assez reconnaissant, et que sa vision de l'idée et de l'actualisation selon l'idée était si séduisante, notamment dans son habillage mathématique, qu'elle m'a incité non seulement à la réflexion, à la recherche et au dialogue critique, comme toute idée importante, mais, de façon plus surprenante et plus pertinente ici, à un effort accru de documentation mathématique », SALANSKIS, 1998, p 172. 
notions d' « Idée dialectique » de « différentielle » et de théorie des « problèmes », de double aspect des « Idées-problèmes » (transcendance et immanence), dans un tressage signifiant avec les travaux de Georges Bouligand (épistémologie du « problème » et de la « différence » en mathématiques), de Louis Rougier (sur les concepts d' " intensité », de « dissymétrie », et à nouveau de « différence »), d'Arend Heyting (sur la « distance » ou « différence logique et mathématique » selon Georges François Cornelis Griss), ou de Paulette Destouches-Février ( « différence et négation en logique », " mathématique et physique »). Cette attention passera de façon massive et décisive dans l'œuvre de Gilles Châtelet qui se forma, en philosophie, auprès de Deleuze ${ }^{13}$.

\section{DU FILON HEIDEGGÉRIEN...}

Pourtant, au moins une occurrence majeure pour le contexte de la philosophie française contemporaine aurait dû faire injonction aux «philosophes professionnels » d'aller y voir au plus près : celle de Martin Heidegger convoqué, dans une économie décisive que nous allons tenter de restituer, dans le texte de 1939, Nouvelles recherches sur la structure dialectique des mathématiques ${ }^{14}$, publié dans les « Actualités scientifiques et industrielles. Essais philosophiques » (le titre de la collection était dû à son directeur, Cavaillès en personne). Il est vrai que Heidegger y est encadré par André Weil sur la généralisation des fonctions abéliennes et par Erich Hecke sur la théorie algébrique des nombres !

L'analyse philosophique de ce texte induit pourtant, à notre avis, un faisceau de questions essentielles à l'élucidation :

1) de l'économie interne du corpus lautmanien comme tel ;

2) du rapport de concors-discors avec le dispositif théorique de Cavaillès ;

3) du trop célèbre « platonisme » de Lautman, dont l'élucidation demande une analyse fine et induit à son tour un effet de feedback sur le totus de la philosophie des philosophes comme telle.

Trois motifs qui nous ont paru suffisants pour en faire un foyer d'analyse relativement autonome.

1. Contrairement à Catherine Chevalley qui, à la fin de son article sur « Albert Lautman et le souci logique », ne consacre en tout et pour tout qu'une ligne relativement « expéditive » à ce recours heideggérien, nous croyons plus juste de tenter une analyse des nécessités internes au texte lautmanien, de cet appel au Vom Wesen des Grundes du

13. À propos de Bouligand, voici ce que nous qualifierons volontiers de « révélation incidente» sur ses liens à Cavaillès. Dans un cours manuscrit reprographié et complètement méconnu, l'auteur annonce dans son avertissement : «Ce fascicule réunit mes notes, établies en vue de la préparation de six conférences, faites en mars-avril-mai 1943 aux étudiants de la Faculté des Lettres de Paris, sur l'invitation de M. Le Professeur Émile Bréhier, Directeur d'études en Philosophie [...]. Quelques étudiants éprouveront certainement le désir d'approfondir [1]es questions [traitées ici]. [...] [L]es thèses de M. Cavaillès leur apporteront, outre leur riche substance, des indications bibliographiques très larges. Note - L'étude de ce texte doit précéder celle des thèses de M. J. Cavaillès. J'ai mis à profit la rédaction du présent cahier pour préciser, sur quelques points, mes recherches antérieures sur la structure des théories », BouligAND, 1948, p. I.

14. Lautman, 1939. 
philosophe de Fribourg. Pour Chevalley, « [d]ans le texte de 1939, il cherche [...] dans un Heidegger imparfaitement identifié l'idée d'une relation transcendantale de domination des Idées aux théories mathématiques, qui rendrait compte de l' "émanation" de celles-ci par "une sorte de procession" 15 ». Voilà pour nous le paradigme d'un universel poncif!

Restituons maintenant le contexte du dispositif théorique dans lequel le texte de 1939 vient naturellement s'inscrire. Quels sont les acquis de la philosophie lautmanienne de l'unité des mathématiques, au moment où apparaît le spectre heideggérien ou l'une de ses déclinaisons?

Lautman, sur les traces de l'axiomatique hilbertienne, s'inscrit d'emblée dans une conception structurale. Cette conception «substitue à la méthode des définitions génétiques [propres pour Lautman aux théories dinosauriennes du XIX siècle] celle des définitions axiomatiques, et loin de vouloir reconstruire l'ensemble des mathématiques à partir de la logique [Lautman attaque cette fois les protocoles du Wiener Kreis], introduit au contraire, en passant de la logique à l'arithmétique et de l'arithmétique à l'analyse, de nouvelles variables et de nouveaux axiomes qui élargissent à chaque fois le domaine des conséquences ${ }^{16}$ ». Il parle alors d'une synthèse du réel qui «participe à la fois de l'intelligence et de la rigueur logique, sans se confondre ni avec l'un, ni avec l'autre ${ }^{17} \gg ;$ c'est la conception structurale et dynamique :

«La conception structurale et la conception dynamique des mathématiques semblent de prime abord s'opposer : l'une tend en effet à considérer une théorie mathématique comme un tout achevé, indépendant du temps, l'autre au contraire ne la sépare pas des étapes temporelles de son élaboration ; pour la première, les théories sont comme des êtres qualitativement distincts les uns des autres, tandis que la seconde voit en chacune une puissance infinie d'expansion hors de ses limites et de liaison avec les autres, par quoi s'affirme l'unité de l'intelligence ${ }^{18}$. »

Ce sont les théories, et non des concepts isolés, qui doivent être l'objet de la philosophie scientifique. C'est en tant que structurales (mouvement autonome et historique d'élaboration des théories) que les mathématiques réalisent des idées dialectiques à travers lesquelles elles paraissent « raconter, mêlée aux constructions auxquelles s'intéresse le mathématicien, une autre histoire plus cachée, et faite pour le philosophe ${ }^{19}$ ».

2. C'est le passage aux schémas de structure : «Des résultats partiels, des rapprochements arrêtés à mi-chemin, des essais qui ressemblent encore à des tâtonnements s'organisent sous l'unité d'un même thème, et laissent apercevoir dans leur mouvement une liaison qui se dessine entre certaines idées abstraites, que nous proposons d'appeler dialectiques $^{20}$. » «Nous n'entendons pas par Idées des modèles dont les êtres mathématiques ne seraient que des copies, mais au véritable sens platonicien du terme, des

15. Chevalley, 1987, p. 71.

16. LaUtMan, 1977, p. 26.

17. LaUtMan, 1977, p. 26.

18. LaUtman, 1977, p. 26.

19. Lautman, 1977, p. 204.

20. Lautman, 1977, p. 204. 
schémas de structure selon lesquels s'organisent les théories effectives ${ }^{21}$. » Or, chose essentielle pour qualifier « autrement » ce « véritable » platonisme :

« [1]a réalité mathématique ne réside [...] pas dans les différences qui sépareraient les être achevés des êtres inachevés, les êtres parfaits des êtres imparfaits; elle réside plutôt dans la possibilité de déterminer les uns à partir des autres, c'est-à-dire dans la théorie mathématique où s'affirment ces liaisons. On voit ainsi que la réalité en question n'est pas celle d'êtres statiques, objets de pure contemplation; s'il existe dans les mathématiques des distinctions qualitatives, elles caractérisent les théories et non les êtres $^{22}$. »

On verra plus loin la solidarité de cette « désubstantialisation », de cette volonté anhyposthatique avec la position de Heidegger.

Ces « schémas de structure » établissent, comme dans toute dialectique, des liaisons spécifiques entre notions contraires : local/global, intrinsèque/extrinsèque, essence/existence, continu/discontinu, fini/infini, algèbre/analyse, etc. Ici, intervient un dispositif fondamental de stratification qui vient déjà «compliquer», bouger, «brouiller » et «ébranler » une vision dialectique platonicienne classique :

«On peut définir la nature de la réalité mathématique de quatre points de vue différents : le réel, ce sont tantôt les faits mathématiques, tantôt les êtres mathématiques, tantôt les théories et tantôt les Idées qui dominent ces théories. Loin de s'opposer, ces quatre conceptions s'intègrent naturellement les unes dans les autres : les faits consistent dans la découverte d'êtres nouveaux, ces êtres s'organisent en théories et le mouvement de ces théories incarne le schéma des liaisons de certaines Idées ${ }^{23}$. »

Ici, les «schémas de structure » constituent avec les faits, les êtres et les théories mathématiques, une quatrième couche du réel mathématique. Or, point central, qui indique déjà pour nous un écart notable avec ce que l'on définit généralement comme "platonisme », « la compréhension des Idées de cette Dialectique se prolonge nécessairement en genèse de théories mathématiques effectives».

« Cherchant à déterminer la nature de la réalité mathématique, nous avons montré [...] que l'on pouvait interpréter les théories mathématiques comme une matière de choix destinée à donner un corps à une dialectique idéale. Cette dialectique semble constituée principalement par des couples de contraires et les Idées de cette dialectique se présentent dans chaque cas comme le problème des liaisons à établir entre notions opposées. $L a$ détermination de ces liaisons ne se fait qu'au sein des domaines où la dialectique s'incarne ${ }^{24}$. »

21. LaUtMan, 1977, p. 204. Ici, ce que Lautman reprend sous le terme d' « Idée », c'est-à-dire, dans une meilleure traduction du grec, sous le concept de «Forme», doit bien plutôt faire signe vers le concept opératoire, «schématisant » et «structurant » de forme différentielle suivant la théorie dite des «p-formes » que vers une «Idée » scolairement, traditionnellement et faussement entendue comme hypostase «platonicienne ».

22. Lautman, 1977, p. 138.

23. LaUtman, 1977, p. 135.

24. Lautman, 1977, p. 253. 
Ainsi, dialectique du concept et mathématiques qui lui donnent corps entretiennent un rapport d' « exclusion interne » selon une étrange logique qui reste encore, aujourd'hui, à interroger : «[...] les théories mathématiques se développent par leur force propre, dans une étroite solidarité réciproque et sans référence aucune aux Idées que leur mouvement rapproche. » Il semble que l'on s'éloigne toujours plus d'un « platonisme » de bon aloi, s'orientant bien plutôt vers un dispositif que Châtelet qualifiait, pour son propre compte, de «force de l'ambiguïté » et de «balances dialectiques ${ }^{25}$.

3. On est alors conduit naturellement à la philosophie des « problèmes ». Les Idées dialectiques sont purement problématiques ; c'est pourquoi :

«Les schémas logiques [les idées travaillant les théories] ne sont pas antérieurs à leur réalisation au sein d'une théorie ; il manque en effet, à ce que nous appelons [...] l'intuition extra-mathématique de l'urgence d'un problème logique, une matière à dominer pour que l'idée de relations possibles donne naissance au schéma de relations véritables ${ }^{26}$.»

Un « problème » n'a de sens que dans une théorie ; ainsi, analysant L'Idéal scientifique des mathématiciens de Pierre Boutroux, Lautman affirme qu'il a tort de dire qu'il y a une "indépendance des êtres mathématiques par rapport aux théories où ils sont définis 27 ». En parlant du « vêtement logique ou algébrique sous lequel nous cherchons à représenter un tel être ${ }^{28} »$, Boutroux présuppose une sorte de neutralité $d u$ formalisme par rapport au sens. Or, l'algèbre moderne montre comment les propriétés des êtres mathématiques peuvent varier avec le domaine dans lequel on les considère. L'introduction de la méthode axiomatique dans les mathématiques rend donc au contraire tout à fait impossible d'isoler des « faits mathématiques » élémentaires, qui seraient comme des briques de construction. Cela suffit, à notre avis, pour saisir ici la détermination par Lautman d'une dialectique des Idées fort atypique, pour ne pas dire atopique. Nous allons voir maintenant comment cet écart tendra à déporter, dans un certain « sens heideggérien », le concept même de « vérité » métaphysique.

4. Le filon heideggérien s'insinue dès lors comme question du passage de l'essence à l'existence. Ce « passage », « prolongement d'une analyse de l'essence en genèse des notions relatives à l'existant ${ }^{29} \gg-$ et donc, transformation de la compréhension d'un sens en genèse d'objets - reprend ici la différence ontologique heideggérienne entre Être et étant. Pour d'évidentes raisons de place, nous nous en tiendrons simplement à quelques premiers frayages d'un filon qu'il restera à creuser. Soucions-nous simplement du « souci » lautmanien :

«L'ordre impliqué par la notion de genèse [lien intime entre la transcendance des Idées et l'immanence de la structure logique de la solution d'un problème au sein des mathématiques] n'est pas [...] l'ordre de la reconstruction logique des mathématiques, au sens où des axiomes initiaux d'une théorie découlent toutes les propositions de la théorie,

25. ChÂTelet, 1993, en particulier « Géométrie et dialectique », « Articuler et engendrer », « La dialectique intensif/extensif ».

26. LaUtMan, 1977, p. 142.

27. LaUTMAN, 1977, p. 139.

28. Boutroux, 1920, cité in Lautman, 1977, p. 139.

29. LaUtMan, 1977, p. 206. 
car la dialectique ne fait pas partie des mathématiques, et ses notions sont sans rapport avec les notions primitives d'une théorie. [...] L'antériorité de la Dialectique [est] celle du "souci" ou de la "question" par rapport à la réponse. Il s'agit là d'une antériorité “ontologique" pour reprendre l'expression de Heidegger, exactement comparable à celle de l' "intention" par rapport au "dessein" 30 . »

Or, détail à notre connaissance jamais relevé par les critiques, ce « souci » (à rapprocher de la Sorge heideggérienne), Lautman l'avait déjà introduit dans la conclusion de sa thèse parue deux ans plus tôt, en 1937 :

«Le seul élément a priori que nous concevions est donné dans l'expérience de cette urgence des problèmes, antérieure à la découverte de leurs solutions [...]. Nous entendons cet a priori dans un sens purement relatif, et par rapport aux mathématiques ; c'est uniquement la possibilité d'éprouver le souci d'un mode de liaison entre deux idées et de décrire phénoménologiquement ce souci, indépendamment du fait que la liaison cherchée peut être ou n'être pas opérable. Certains de ces "soucis" logiques se trouvent dans l'histoire de la philosophie, comme par exemple le souci des liaisons entre le même et l'autre, le tout et la partie, le continu et le discontinu, l'essence et l'existence ${ }^{31}$. »

Martin Heidegger, ici, n'est pas convoqué comme tel : et pourtant, c'est déjà de lui dont il s'agit, par quelque chose qui lui est comme télépathiquement, c'est-à-dire anticipativement promis, au nom de la mathématique, et ce dans l'attente conjuguée d'un mode inouï et radical de questionnement de la métaphysique occidentale :

«[...] mais les théories mathématiques pourront inversement faire naître l'idée de problèmes nouveaux qui n'auraient pas été formulés abstraitement auparavant. La philosophie mathématique, telle que nous la concevons, ne consiste donc pas tant à retrouver un problème logique de la métaphysique classique au sein d'une théorie mathématique, qu'à appréhender globalement la structure de cette théorie pour dégager le problème logique qui se trouve à la fois défini et résolu par l'existence même de cette théorie ${ }^{32}$. »

Revenons une dernière fois au texte de 1939, où Heidegger est commenté ad nominem et parfaitement « identifié 33 ». Non seulement Lautman réinvestit le dispositif heideggérien de la « différence ontologique » (de l'être et de l'étant), affirmant que les Idées dialectiques sont aux théories mathématiques ce que l'être et le sens de l'être sont à l'étant et à l'existence de l'étant, mais, point ici capital, il mobilise dans le même geste la catégorie heideggérienne de vérité dépliée comme « dévoilement de l'être »:

30. LaUtMan, 1977, p. 210.

31. LaUtMan, 1977, p. 142.

32. LAUTMAN, 1977, p. 142-143. Émettons, par provision, l'hypothèse d'un lien à effectuer au rapport homologie-cohomologie, et en particulier au théorème de Whitney montrant l'équivalence ou les «morphismes » de deux théories (ou dispositifs).

33. Notons que cette «identification », il la revendiquera jusqu'à la fin, en particulier face au philosophe : «M. Hyppolite me dit que poser un problème, c'est ne rien concevoir ; je lui réponds, après Heidegger, que c'est déjà délimiter le champ de l'existant» - «Réponse à Hyppolite » à la séance de la Société française de philosophie du 4 février 1939, in CAVAILlĖs, 1994, p. 630. 
cette a-léthéia que le philosophe de Freiburg traduit en sa propre langue par Entbergung (la « découverture ») :

« Il arrive alors, et cela est pour nous le point fondamental, que ce dévoilement de la vérité ontologique de l'être ne puisse se faire, sans que se dessinent en même temps les aspects concrets de l'existence ontique: "Caractéristique entre autres degrés est, par exemple, le projet qui en esquissant la constitution de l'être de l'existant, jalonne en même temps un champ déterminé (Nature, Histoire) comme le domaine où il sera possible à une connaissance scientifique de constituer des objets" (Vom Wesen des Grundes, tr. fr. par Henry Corbin, in Qu'est-ce que la métaphysique ?, Gallimard, Paris, 1938, p. 57). On voit donc, d'après ce texte, une même activité se dédoubler, ou plutôt agir sur deux plans différents : la constitution de l'être de l'existant, sur le plan ontologique, est inséparable de la détermination, sur le plan ontique, de l'existence en fait d'un domaine où prennent vie et matière les objets d'une connaissance scientifique. Le souci de connaître ce qui fait l'essence de certains concepts n'est peut-être pas orienté primitivement vers les réalisations de ces concepts, mais il se trouve que l'analyse conceptuelle aboutisse nécessairement à projeter comme au-devant du concept, les notions concrètes en lesquelles il se réalise ou s'historialise ${ }^{34}$. »

Or chose absolument remarquable, quelques lignes plus bas Lautman va jusqu'à saisir certaines faiblesses de ce que l'on a pu qualifier depuis comme le «premier Heidegger» (celui de «l'analyse existentiale ») radicalisant ainsi, ante litteram, la propre auto-« critique » heideggérienne de Heidegger :

« La distinction de l'essence et de l'existence, et surtout le prolongement d'une analyse de l'essence en genèse des notions relatives à l'existant, sont quelquefois masqués dans la philosophie de Heidegger par l'importance des considérations existentielles, relatives à l'Être-dans-le-Monde, telles qu'elles apparaissent dans Sein und Zeit ${ }^{35}$. »

On pourrait parler ici d'une remarquable perspicuitas anticipative... C'est cette conscience, certainement aiguisée par ses propres intérêts, qui lui a permis de si bien saisir les propos et l'enjeu heideggériens. Le Wesen vom Grundes (auquel renvoie Lautman) opère comme principe épochal qui entre ainsi dans la suite des établissements successifs par lesquels de nouveaux champs d'intelligibilité se sont fait jour, brusquement et imprévisiblement, depuis les débuts de notre histoire. «Époque » ne veut pas dire ici un intervalle de temps dans le cours de ce qui arrive, mais le trait fondamental de l'envoi : la retenue dans chaque cas, grâce à laquelle ce qu'il y a peut être perçu. Le surgissement d'un principe épochal n'est rien d'autre que l'arrivée de l'économie concrète d'un âge métaphysique. Un principe épochal se manifeste à la manière d'une adresse, d'une réquisition. Il requiert une communauté d'hommes en vue de modes de pensée et d'action finis.

Les conséquences de cette inscription dans le sillon heideggérien sont fondamentales pour une juste restitution de cette pensée et de cette philosophie lautmaniennes dans ce qu'elles conservent de plus actuel :

34. LaUtman, 1977, p. 206.

35. Lautman, 1977, p. 206-207. Voir HeideGGER, 1927. 
1. Si pour Lautman, exposer le sens philosophique des mathématiques consiste à montrer leur « rattachement à une métaphysique (ou Dialectique) dont elles sont le prolongement nécessaire », il est maintenant évident qu'il ne s'agit pas de n'importe quelle «métaphysique », et certainement pas de cette métaphysique « classique » qui caractérise plutôt celle, déjà éculée et de surcroît aveugle, du « Cercle de Vienne »; son engagement se fait désormais aux côtés du questionnement le plus radical de cette « métaphysique classique » dont Heidegger, comme on le sait, entamait ce qu'il appela «le dépassement » ou la Destruktion ${ }^{36}$.

2. Son alignement sur l'interprétation heideggérienne de l'a-léthéia conçue comme « dévoilement de l'être » devrait radicalement disqualifier son trop fameux «platonisme »-du moins au sens que l'on lui attribue traditionnellement avec une bien trop grande désinvolture. Ainsi, sur ce point, Cavaillès lui-même aura donné d'une certaine façon le mauvais exemple :

«Dans les discussions proprement mathématiques qui avaient lieu entre tenants de l'École de Vienne et de l'École de Hilbert, on se posait la question de savoir s'il y avait une région d'objets idéaux auxquels pourraient se référer les mathématiques - on appelait cela un platonisme, je crois que l'expression ne correspond pas très bien à la chose, mais peu importe le mot $^{37}$. »

Voilà pour la désinvolture ! Ce qu'il engage avec la théorie heideggérienne de la vérité, c'est le renoncement à toute conception du vrai saisi comme omoiosis ou comme adaquatio rei et intellectu, version «moderne » et cartésienne de l'idéalisme platonicien. La question n'est plus celle de l' « adéquation de l'Idée au Réel », point sur lequel Lautman n'a eu de cesse de revenir (voir entre autres sa critique de Boutroux).

3. L'engagement dans un autre concept de la vérité par un «dépassement» de la métaphysique est solidaire d'une déconstruction des schèmes substantialistes. Pour Heidegger, la recherche métaphysique d'un fondement premier est par définition à l'affût de quelque chose qui se tienne en-dessous des phénomènes, à l'affût d'un hypokémeinon, c'est-à-dire d'un « substrat ». Or Lautman est rigoureusement engagé dans un procès de désubstantialisation de la philosophie mathématique, en particulier par un déplacement et une complicatio du rapport métaphysiquement fondatif forme/matière.

À titre d'exemple, et parmi tant d'autres, toujours de l'année1937 : le théorème de Jacques Herbrand sur les « champs » s'offre pour Lautman comme un cas presque pur de solidarité entre un ensemble d'opérations formelles définies par un système d'axiomes et l'existence d'un domaine où ces opérations sont réalisables. Il note à ce propos :

« Il semble qu'une certaine restriction adhère encore à ce schéma logique ; la genèse n'y a lieu en effet que dans un sens, des opérations au domaine. Or, si l'on peut établir

36. Il faut rapprocher ceci d'une autre «métaphysique» de mathématicien, celle revendiquée et partagée par son ami André Weil : «Rien n’est plus fécond, tous les mathématiciens le savent, que ces obscures analogies, ces troubles reflets d'une théorie à une autre, ces furtives caresses, ces brouilleries inexplicables ; rien aussi ne donne plus de plaisir au chercheur. Un jour vient où l'illusion se dissipe ; le pressentiment se change en certitude ; les théories jumelles révèlent leur source commune avant de disparaître ; comme l'enseigne la Gita, on atteint à la connaissance et à l'indifférence en même temps. La métaphysique est devenue mathématique, prête à former la matière d'un traité dont la beauté froide ne saurait plus nous émouvoir », WEIL, 1980, p. 409.

37. CAvaillès, 1994, p. 603. 
entre le domaine et les opérations définissables sur lui, une appropriation rigoureuse, on peut chercher à déterminer aussi bien les opérations à partir du domaine que le domaine à partir des opérations [...]. Notre intention étant de montrer que l'achèvement interne d'un être s'affirme dans son pouvoir créateur, cette conception devrait peut-être logiquement impliquer deux aspects réciproques : l'essence d'une forme se réalisant au sein d'une matière qu'elle créerait, l'essence d'une matière faisant naître les formes que sa structure dessine. [...] En fait, le schéma des genèses que nous allons décrire au sein de théories plus compliquées, abandonne l'idée trop simpliste de domaines concrets et d'opérations abstraites qui posséderaient en eux-mêmes comme une nature de matière ou une nature de forme ; cette conception tendrait en effet à stabiliser les êtres mathématiques dans certains rôles immuables et ignorerait le fait que les êtres abstraits qui naissent de la structure d'un domaine plus concret peuvent à leur tour servir de domaine de base pour la genèse d'autres êtres ${ }^{38}$. »

On parlera ici du résultat d'une certaine axiomatique philosophique et de son autoapplication par «réflexion »: ce qu'affirme Lautman de la logique mathématique, des théorèmes d'existence dans la théorie des fonctions algébriques, ou de la théorie de la représentation des groupes en tant que domaines différents de l'investigation transcendantale, est « retourné » par symétrie sur le dispositif philosophique lui-même, qui, en un premier temps, se trouve situé en position d'opération - et inversement. C'est là un opérateur d'interaction dialectique extrêmement puissant qui, à partir du domaine de la physique-mathématique, sera inducteur d'effets dans le champ et l'activité philosophiques.

Destruktion de la métaphysique, «déconstruction » de la Vérité conçue comme omoiosis, désubstantialisation : Heidegger l'aura lui-même (partiellement) saisi, à la même époque, dans le champ de prédilection des enquêtes lautmaniennes - la physique mathématique. C'est toute la question de ce qui pourrait s'intituler : Heidegger et la Quantique.

\section{... AU NEUD « INVOLUTIF » DE LA PHYSIQUE MATHÉMATIQUE}

Le lieu le plus impressionnant où l'approche lautmanienne apparaît comme quasi « prophétique », le domaine de sa plus grande inspiration philosophique et intuitive, le couplage dialectique sur lequel viennent se polariser toutes les promesses de sa philosophie mathématique, semblent sans conteste toucher à la problématique de « la symétrie et de la dissymétrie en mathématiques et en physique ${ }^{39} \gg$.

Ce texte pourrait être emblématique de l'écart du binôme Cavaillès-Lautman. Si dès sa thèse Lautman avait déjà montré son intérêt pour la physique mathématique - en particulier par son étude attentive des textes d'Élie Cartan (sur la « généralisation de la

38. LaUtman, 1977, p. 32.

39. C'est le titre d'un des tout derniers textes de Lautman (1942), d'abord imprimé en fascicule séparé dans les « Actualités scientifiques et industrielles » en 1946, avant de rejoindre d'autres contributions dans le projet initié en 1942 par François LE LiOnNAIS, Les Grands Courants de la pensée mathématique, publié en 1948 (voir LE LiOnNAIS, 1998, la nouvelle édition réalisée à l'initiative de notre ami mathématicien Bernard Teissier). 
notion d'espace », « le parallélisme absolu et la théorie unitaire ») ou de ceux d'Hermann Weyl (sur les « espaces riemanniens »), mais également par sa lecture de l'ouvrage d'Arthur Eddington, Espace, temps, gravitation ${ }^{40}$, ou par celle de La Structure des nouvelles théories physiques de Gustave Juvet ${ }^{41}$-, c'est sur la fin de sa vie, avant de tomber au combat, qu'il orienta toute son activité épistémologique sur ces questions de physique (et ce, grâce à son exceptionnel bagage mathématique). Son apport très personnel et extrêmement original touche principalement aux questions d'enveloppement des notions de symétrie et de dissymétrie. Il opère son amorce thématique par une analyse du travail pionnier de Louis Pasteur sur la dissymétrie cellulaire par « énantiomorphie », « à l'origine de toutes les théories structurales de la stéréochimie contemporaine ${ }^{42}$ ». Puis il passe aux travaux fondateurs en physique de Pierre Curie :

« [...] le mélange de symétrie et de dissymétrie devient pour lui condition nécessaire du phénomène physique en général [...]. A tout phénomène physique est liée l'idée d'une saturation de la symétrie, d'une symétrie maxima compatible avec l'existence de ce phénomène et qui le caractérise. Un phénomène ne peut exister que dans un milieu possédant sa symétrie caractéristique ou une symétrie moindre. Si donc on appelle élément de dissymétrie l'absence d'un élément de symétrie, on conçoit comment Pierre Curie a pu écrire: "Certains éléments de symétrie peuvent coexister dans certains phénomènes, mais ils ne sont pas nécessaires. Ce qui est nécessaire, c'est que certains éléments de symétrie n'existent pas. C'est la dissymétrie qui crée le phénomène" (P. Curie, «Sur la dissymétrie dans les phénomènes physiques », CEuvres complètes, p. 126) ${ }^{43}$. »

Lautman enchaîne cette idée de « symétrie limite » au Timée de Platon, et en particulier à sa théorie du réceptacle conçu comme « lieu » qu'est la Chora. Or, il ne s'agit pas ici (et c'est là la force spéculative prodigieuse de Lautman) d'une référence d'apparat :

«Cette référence à Platon permet de comprendre que les matériaux de l'Univers ne sont pas tant les atomes et les molécules de la théorie physique que ces grands couples de contraires idéaux comme le Même et l'Autre, le Symétrique et le Dissymétrique, associés entre eux selon les lois d'un harmonieux mélange [...]. Platon suggère également davantage [...]. Les propriétés du lieu et de la matière ne sont pas chez lui purement sensibles ; elles sont [...] la transposition géométrique et physique d'une théorie dialectique. Il se pourrait de même que la distinction de la gauche et de la droite, telle qu'on l'observe dans le monde sensible, ne soit que la transposition sur le plan de l'expérience d'une symétrie dissymétrique constitutive également de la réalité abstraite des mathématiques ${ }^{44}$. »

40. EDDington, 1921.

41. JUVET, 1933.

42. Lautman, 1977 , p. 240.

43. LaUtman, 1977, p. 240. « Chaque physicien se sert journellement, d'une façon plus ou moins explicite, de ces notions de symétrie ; aussi sommes-nous fort étonnés de ne les voir énoncées dans aucun traité de Physique. Ces notions sont cependant fondamentales et, énoncées dès le début, elles facilitent beaucoup aux élèves la compréhension des phénomènes. Bien des démonstrations se trouvent en effet immédiatement simplifiées lorsqu'on fait intervenir les notions de symétrie », CuRIE, 1908, p. 150.

44. Lautman, 1977, p. 241. 
Ici, à l'apogée de sa force de conviction, c'est comme si Lautman faisait fulgurer le geste de sa pensée par un pivotement au cœur même du domaine ou du champ, à la fois thématique et opérationnel de la physique mathématique. C'est ici qu'elle s'illustre comme dans l'épure d'un pur diagramme de pensée, où la puissance virtuelle de ses schèmes viendrait se visualiser dans l'œil de l'esprit à la fois mathématicien et philosophe. La pensée s'accorde quelque chose comme sa propre perception « scopique » de soi 45 . C'est au moment même où il investit concrètement le domaine physico-mathématique que se marque la toute-puissance de son dispositif dialectique. La philosophie vient ici révéler son habitation de/dans la Science, révélant ainsi leur double pouvoir de suggestion réciproque : car ici, la Science pense (et se pense), comme habitée par ses spectres philosophiques. C'est dans un tel moment de suspens, dans cet «entre-deux Mondes » que viennent d'elles-mêmes s'annuler les objections factices élevées contre un prétendu « arbitraire » de sa Dialectique. C'est comme si l'opération dialectique se découvrait d'un coup, se rendait subitement lisible à l'œil de la théorie par sa propre «montée vers l'absolu », lui procurant ainsi une sorte de « surface universelle de recouvrement».

Ce sentiment ne fait dès lors que s'aiguiser avec le déroulement de l'argumentation lautmanienne. Par un dernier parcours exemplifiant, et en forme d'insistance, Lautman dégage le foyer mathématique de l'entier dispositif : l'opération d'involution posée comme opérateur « universel » et noyau de toute structure duale (ou principe de « dualité ») - une notion qui est abordée de manière identique par Weyl dans le cas de la transformation appelée automorphisme pour un espace «zero-dimensionnel» (c'est-à-dire réduit à la structure du point) :

«Il nous faut insister un instant sur la façon dont la distinction de la gauche et de la droite dans le monde sensible peut symboliser la non-commutativité de certaines opérations abstraites de l'algèbre. La propriété fondamentale de la symétrie par rapport à un plan, c'est qu'opérée une fois, elle donne une figure distincte par son orientation de la figure primitive, et que répétée une deuxième fois, elle redonne la figure primitive. C'est en ce sens que la symétrie est dite opération involutive. Considérons maintenant une opération algébrique portant sur deux quantités $\mathrm{X}$ et $\mathrm{Y}$, et que nous écrivons (XY), la parenthèse pouvant désigner un produit ordinaire, ou tout autre opération définie sur les deux variables. C'est une opération non commutative si $(X Y) \neq(Y X)$ et la noncommutativité la plus féconde en mathématiques est celle où l'on a $(X Y)=-(Y X)$. L'opération (XY) est dissymétrique en $\mathrm{X}$ et $\mathrm{Y}$, mais on vérifie facilement qu'elle définit une opération involutive comme la symétrie ordinaire. Les expressions (XY) et (YX) sont dites antisymétriques et ce mot traduit bien le mélange de symétrie et de dissymétrie qui est ainsi profondément installé au cœur de l'algèbre contemporaine. Toute la théorie des groupes continus de Lie repose sur la non-commutativité du produit de deux opérations infinitésimales du groupe, et c'est cette théorie qui, étroitement associée à la théorie des formes de Pfaff, expressions à multiplication antisymétrique, a permis à M. Cartan de découvrir une profonde analogie entre les espaces de Riemann généralisés qui interviennent dans les théories physicogéométriques de la Relativité, et l'espace des groupes de $\mathrm{Lie}^{46}$.»

45. Sur ce statut de la «métaphore à la lettre », voir AlunNI, 2001.

46. LautMan, 1977, p. 244. 
Working physicists et working mathematicians devraient aujourd'hui encore prendre exemple sur ce texte, tant pour sa remarquable clarté que pour la lucidité à la fois « structurale » et définitionnelle qu'il dégage.

Passant à la théorie des particules de la mécanique ondulatoire broglienne, Lautman postule que «l'antisymétrie semble jouer dans la Nature un rôle beaucoup plus fondamental que la symétrie [...] ; elle joue un rôle capital dans l'explication du lien moléculaire ${ }^{47} \gg$. Après une analyse fine de l'antisymétrie de spin, le rabattement de la distinction des fonctions d'ondes à son fondement mathématique «d'une dissymétrie interne du groupe de permutations », Lautman opère sa « montée vers l'absolu », c'està-dire ici « vers le principe de dualité » 48 :

« Transposée dans un langage plus abstrait, cette situation équivaut à la possibilité de distinguer au sein d'un même être deux êtres distincts $\mathrm{X}$ et $\mathrm{X}$ ' qui seront dits en dualité, d'une part si l'on peut définir pour chacun d'eux une orientation ou une ordonnance inverse de celle de l'autre, et d'autre part s'il existe une relation involutive entre eux, c'est-à-dire si X est à $X^{\prime}$ ce que $X^{\prime}$ est à $X$, c'est-à-dire si $\left(X^{\prime}\right)^{\prime}=X^{49}$. »

Enfin, parcourant à nouveau les étapes fondamentales de la constitution de la catégorie de « dualité » (algèbre de George Boole, géométrie projective de Jean Victor Poncelet), Lautman conclut sur un exposé des recherches les plus récentes menées alors en algèbre abstraite (George Birkhoff, John von Neumann, Valere Glivenko et Oysten Ore) à travers la «théorie générale des structures » (théorie des réseaux - ou lattices en anglais -, dont on sait aujourd'hui toute l'importance, jusqu'en théorie des catégories), introduisant la notion de « dual » (qu'il appelle « duel ») et celle d'anti-isomorphisme :

«La théorie générale des structures repose donc sur la possibilité de structurer de deux façons inverses l'une de l'autre un même ensemble, et c'est pour nous un résultat d'une importance philosophique capitale de voir cette dualité interne de deux êtres anti-

47. Lautman, 1977, p. 245-246.

48. La dualité (qui est une propriété mathématico-physique fondamentale) possède des termes analogues tels que ceux de « conjugaison » ou de « réciprocité » (ce qui renvoie globalement à l'idée centrale de «symétrie »). C'est une notion qui figure dans la dénomination tant de constructions générales (« dualité » des espaces linéaires, foncteurs « adjoints » en théorie des catégories), que de théorèmes particuliers ( « dualité » d'Henri Poincaré ou lois de « réciprocité » d'Emil Artin). Dans toutes les utilisations variées du terme est contenue l'idée de symétrie bilatérale d'un objet, d'une construction ou d'une théorie mathématique. En fait, l'idée de dualité possède un caractère extrêmement général. Parmi les corrélations les plus proches de la dualité mathématique, on notera par exemple : le principe de classifications « dichotomiques » (en philosophie, le « dualisme » opposé au « monisme » est aussi vieux que la philosophie elle-même : c'est par exemple l'enjeu du dispositif de « déconstruction » à l'œuvre dans la philosophie derridienne); les «oppositions» du structuralisme contemporain; la « complémentarité » en physique quantique, modélisée par les inégalités de Heisenberg - certains couples «séparés» de grandeurs classiques se trouvent fonctionner ici en variables conjuguées ( « complémentaires»). Ces inégalités estiment alors la production d'une «dispersion» autour de la valeur moyenne de ces deux observables qui ne commutent plus. Le « prototype » de la dualité mathématique est sans conteste celle des espaces linéaires. On pourrait même aller jusqu'à dire que, par elle, s'y constitue le statut ontologique de la linéarité, dont les conséquences physiques sont " phénoménales ». Dans ce cadre, et à titre d'exemple élémentaire, l'abaissement ou l'élévation des indices (ainsi que la contraction) sont lus comme des opérations de dualisation linéaire d'opérations tensorielles (voir sur cette catégorie essentielle, et transdisciplinaire, l'article remarquable de GEL'FAND et MANIN, 1978).

49. Lautman, 1977, p. 247. 
symétriques, distinguables au sein d'un même être, former le principe générateur d'une immense moisson de réalité mathématique ${ }^{50}$. »

Comment ne pas penser ici à l'ombre projetée des Récoltes et semailles d'Alexandre Grothendieck?

Pour conclure, mais en forme d'ouverture, on sait aujourd'hui toute l'importance cardinale des questions et des techniques investies par Lautman : combien a tenu ses promesses le «non-commutatif» (il n'est qu'à penser à l'œuvre d'Alain Connes sur la géométrie non-commutative) ; combien la notion de « dualité » et de structure « duale », que Lautman avait abordées, dès sa thèse de 1937, en travaillant les «théorèmes de dualité » définis comme "schémas de structure », et dans le cadre déjà parlant des « propriétés intrinsèques et des propriétés induites », combien ces notions sont devenues le cœur même des problématiques physico-mathématiques les plus actuelles. On sait, par exemple, l'importance des «théorèmes de dualité » dans la caractérisation des algèbres de Hopf pour le développement des groupes quantiques. Last, but not least, notons que la fameuse « différence ontologique » est elle-même définie par Heidegger comme «Dualité » (Zwiespalt) !

Pourtant, les idées de Lautman et de Weyl sur ce point ne retiendront pratiquement pas l'attention avant... 1956. En ce qui concerne l'antagonisme « local-global », Lautman avait également su faire appel à l'œuvre d'Élie Cartan, que bien peu appréciaient à sa valeur avant 1935, et dont la place centrale dans toutes les mathématiques est maintenant universellement reconnue. Et nous ne parlerons pas ici de ce qui constituait déjà son attirance pour ce qui deviendra la fascinante théorie des catégories.

Ainsi, c'est un mathématicien, et pas des moindres, qui soutient :

« On voit donc que Lautman avait pressenti cet extraordinaire développement de la mathématique, auquel le destin ne lui a pas permis d'assister ; il l'eut rempli d'enthousiasme, tant par la moisson inégalée de théories nouvelles et de solutions d'anciens problèmes, que par le caractère éminemment esthétique qu'offrent maintenant (à ceux qui comme Lautman, cherchent à les comprendre) les parties centrales de cet immense édifice ${ }^{51}$. »

Nous dirons enfin que ce qui fait l'écart (inversement proportionnel à leur notoriété dans le milieu philosophique français) avec son ami Jean Cavaillès (mais c'est ici une question de sensibilité personnelle dans l'approche de la recherche, et donc une question de style), c'est l'instauration par Lautman d'une «philosophie ouverte ${ }^{52}$ » qui soit véritablement tournée vers les promesses de ce qui n'était alors encore qu'un futur : celui entrelacé de la philosophie, de la mathématique, de la physique (et de la physiquemathématique), c'est-à-dire rien de moins que notre propre présent.

Charles ALUNNI (novembre 2000).

50. LaUtman, 1977, p. 250.

51. Voir l'avant-propos de Jean Dieudonné, in Lautman, 1977, p 19.

52. BACHELARD, 1940, p. 67 : «Finalement la philosophie de la science physique est peut-être la seule philosophie qui s'applique en déterminant un dépassement de ses principes. Bref elle est la seule philosophie ouverte. Toute autre philosophie pose ses principes comme intangibles, ses premières vérités comme totales ou achevées. Toute autre philosophie se fait gloire de sa fermeture. » 


\section{LISTE DES RÉFÉRENCES}

AlunNi (Charles), 2001, «Pour une métaphorologie fractale », Revue de synthèse, ${ }^{\circ}$ spéc. : « Objets d'échelles », t. CXXII, 1, janv.-mars 2001, p. 154-171.

BACHELARD (Gaston), 1938, La Formation de l'esprit scientifique, Paris, Vrin.

Bachelard (G.), 1940, La Philosophie du non. Essai d'une philosophie du nouvel esprit scientifique, Paris, Presses universitaires de France.

BACHELARD (G.), 1950, « L'œuvre de Jean Cavaillès », in FerRIères (Gabrielle), Jean Cavaillès. Un philosophe dans la guerre. 1903-1944. Avec une étude de son æeuvre par Gaston Bachelard, Paris, Presses universitaires de France, p. 221-234.

BAdiou (Alain), 1988, L'Être et l'événement, Paris, Seuil.

BADIOU (A.), 1989, Manifeste pour la philosophie, Paris, Seuil.

BAdIou (A.), 1992, Conditions, Paris, Seuil.

Badiou (A.), 1997, Deleuze. La clameur de l'Être, Paris, Hachette.

Badiou (A.), 1998a, Abrégé de métapolitique, Paris, Seuil.

BADIOU (A.), 1998b, Court traité d'ontologie provisoire, Paris, Seuil.

BENIS-SINACEUR (Hourya), 1987a, « Lettres inédites de Jean Cavaillès à Albert Lautman », Revue d'histoire des sciences, t. XL, 1, p. 117-128.

BenIs-SinACEUR (H.), 1987b, «Lettre inédite de Gaston Bachelard à Albert Lautman », Revue d'histoire des sciences, t. XL, 1, p. 129.

Bouligand (Georges), 1948, Les Méthodes mathématiques. De l'intuition à l'algébrisme. Les structures des théories. L'axiomatisation. Les méthodes directes. La formalisation, nouvelle édition avec des compléments 1) sur la formation et l'évolution des théories mathématiques (rôle des problèmes et de la synthèse) ; 2) sur l'ossature algébrique de la mathématique classique, Paris, Centre de documentation universitaire.

Boutroux (Pierre), 1920, L'Idéal scientifique des mathématiciens dans l'Antiquité et dans les Temps modernes, Paris, Alcan.

CANGUILHEM (Georges) et EHRESMAN (Charles), 1947, « Avertissement des éditeurs à la première édition de Sur la logique et la théorie des sciences », in CAVAILLÈs, 1994, p. 473-560.

CAvaillès (Jean), 1994, Euvres complètes de philosophie des sciences, Paris, Hermann.

ChÂtelet (Gilles), 1993, Les Enjeux du mobile. Mathématique, physique, philosophie, Paris, Seuil.

Chevalley (Catherine), 1987, « Albert Lautman et le souci logique », Revue d'histoire des sciences, t. XL, 1, p. 49-77.

CurIE (Pierre), 1908, Euvres, Paris, Gauthier-Villars.

Deleuze (Gilles), 1968, Différence et répétition, Paris, Presses universitaires de France.

Deleuze (G.), 1969, Logique du sens, Paris, Minuit.

Deleuze (G.), 1980, Capitalisme et schizophrénie. Mille Plateaux, Paris, Minuit.

Deleuze (G.), 1986, Foucault, Paris, Minuit.

Deleuze (G.), 1988, Le Pli. Leibniz et le baroque, Paris, Minuit.

EdDington (Arthur), 1921, Espace, temps, gravitation, Paris, Hermann.

GEL'FAND (Izrail Moiseevich) et MANIN (Yuri), 1978, « Dualità », in Enciclopedia, Turin, Einaudi, vol. V, p. 126-178. 
HEIDEGGER (Martin), 1927, « Sein und Zeit », in Jahrbuch für Philosophie und phänomenologische Forschung, Bd VIII, Halle, Niemeyer.

HeIDEGger (M.), 1938, Qu'est-ce que la métaphysique ?, Paris, Gallimard.

HeidegGer (M.), 1984, Zur Frage nach der Bestimmung der Sache des Denkens, St. Gallen, Erker.

Huisman (Bruno), 1985, « Jean Cavaillès et la philosophie française de l'entre-deux-guerres » in Jean Cavaillès : philosophe, résistant, Amiens, Centre régional de documentation pédagogique, p. 25-33.

Juvet (Gustave), 1933, La Structure des nouvelles théories physiques, Paris, Alcan.

LAUTMAN (Albert), 1939, Nouvelles recherches sur la structure dialectique des mathématiques, Paris, Hermann (Actualités scientifiques et industrielles. Essais philosophiques, 804).

Lautman (A.), 1977, Essai sur l'unité des mathématiques et divers écrits, Paris, Union générale d'éditions.

Le Lionnais (François), 1998, Les Grands Courants de la pensée mathématique, Paris, Hermann.

Pетітот (Jean), 1987, « Refaire le Timée. Introduction à la philosophie mathématique d'Albert Lautman », Revue d'histoire des sciences, t. XL, 1, p. 79-115.

Salanskis (Jean-Michel), 1998, «Pour une épistémologie de la lecture », in JuRdant (Benoît), dir., Impostures scientifiques, Paris, La Découverte/Alliage, p. 157-194.

VAN DER WAERDEN (Bartel Leendert), 1930, Moderne algebra, Berlin, Springer.

WEIL (André), 1980, «De la métaphysique aux mathématiques », in ID., Euvres scientifiques, New York, Springer, vol. II, p. 409-412. 